\title{
Retificação da alumina com rebolo diamantado utilizando-se diferentes métodos de lubri-refrigeração
}

\section{(Grinding of alumina with diamod wheel using different cooling methods)}

\author{
M.C.S.Alves ${ }^{1}$ L. D. Neto ${ }^{1}$,E.C.Bianchi ${ }^{1}$, P.R.Aguiar ${ }^{2}$ H. Fujita ${ }^{3}$, E. J. Silva ${ }^{4}$ \\ ${ }^{I}$ Departamento de Engenharia Mecânica, Universidade Estadual Paulista - UNESP, Bauru, SP \\ ${ }^{2}$ Departamento de Engenharia Elétrica, Universidade Estadual Paulista - UNESP, Bauru, SP \\ ${ }^{3}$ Máquinas Agrícolas Jacto S/A, C.P. 35, R. Dr. Luiz Miranda 1650, Centro, Pompeia, SP \\ ${ }^{4}$ Escola de Engenharia de S. Carlos - EESC-NUMA-IFM, USP, \\ Av. Trabalhador S.-Carlense 400, S. Carlos, SP 13566-590 \\ bianchi@feb.unesp.br
}

\begin{abstract}
Resumo
O sucesso da cerâmica estrutural na maioria das aplicações depende não somente das propriedades do material e do projeto da peça, mas também da qualidade do produto usinado. Um dos fatores citados como obstáculo à ampla utilização de componentes cerâmicos é a sua falta de confiabilidade, em função da grande dispersão dos valores de resistência mecânica provocada por defeitos que podem ter origem no processo de retificação. A retificação de cerâmica, assim como a de aços e superligas, é realizada normalmente com abundância de fluidos de corte. A utilização destes resulta em problemas ecológicos, fisiológicos e econômicos. Desde a última década, a opinião pública, o mercado, as leis e regulamentos têm pressionado as indústrias para considerar os riscos ambientais na manufatura. Com o propósito de avaliar alternativas ao método convencional de lubri-refrigeração, foi estudada a viabilidade da técnica de refrigeração otimizada, onde o fluido de corte é aplicado à mesma velocidade periférica do rebolo, penetrando na região de corte com menos turbulência. Também foi analisada a técnica da Mínima Quantidade de Lubrificação (MQL), na qual o grande volume de fluido de corte é substituído por um jato de ar contendo uma quantidade ínfima de lubrificante. Os métodos foram comparados através da análise da força tangencial de corte, rugosidade e relação G. Concluiu-se ainda que a técnica de MQL mostrou-se inviável na retificação de cerâmica nas condições propostas e que os resultados com a lubri-refrigeração otimizada não permitiram afirmar que esta resultou em melhores valores de rugosidade e relação G quando comparada com à técnica convencional.
\end{abstract}

Palavras-chave: retificação, cerâmica, lubri-refrigeração, MQL.

\begin{abstract}
The success of structural ceramics in most applications depends not only on the materials properties and component design, but also the quality of machined products. One factor usually mentioned as a barrier to the wide use of ceramics is the lack of reliability of ceramic components, because the variability on the mechanical strength caused by defects that can be impaired in the grinding process. The grinding of ceramics, as much as with steel grinding is performed generally with excess of cutting fluids (coolants). The use of coolants results in serious ecological, physiological and economic issues. Since last decade the public opinion, the market, the laws and regulations have pushed the industries to consider the environmental risks in the manufacturing process. Then, in order to evaluate alternatives to the conventional method of cooling and lubrication, it was studied the viability of the optimized technique, where the cutting fluid is applied to the same peripherical speed of the wheel, penetrating in the cutting zone with less turbulence. Also, it was verified the technique of Minimum Quantity of Lubricant (MQL), where a large amount of cutting fluid is replaced by an air compressed flow containing a small quantity of lubricant. The methods were compared through performance analysis of cutting forces, roughness and wheel ( $G$ ratio). As a conclusion is that MQL is not viable in ceramics grinding with the working conditions proposed. The results obtained with optimized technique are insufficient to state this technique presents better roughness and G-ratio values when compared to conventional method.
\end{abstract}

Keywords: grinding, ceramic, cooling, minimum quantity of lubrication $(M Q L)$.

\section{INTRODUÇÃO}

Embora os materiais cerâmicos para aplicações técnicas sejam conhecidos há mais de duzentos anos, as cerâmicas avançadas, cuja composição, microestrutura e propriedades diferem dos materiais cerâmicos tradicionais, começaram a ser desenvolvidas por volta de 1970. Esses materiais foram desenvolvidos para aplicações especiais, onde materiais convencionais como metais ou polímeros não atendiam às solicitações. Devido às suas ligações iônicas e/ou covalentes, 
as cerâmicas são extremamente duras e rígidas e por isso, materiais de excelente resistência ao desgaste à temperatura ambiente ou elevada. Essas propriedades, aliadas à inércia química, as tornam materiais ideais para aplicações de alta tecnologia, como componentes de motores, válvulas, selos mecânicos, sensores de gás, biomateriais, ferramentas de corte, rolamentos, componentes eletrônicos e materiais para aplicações aeroespaciais.

O processo de fabricação de componentes cerâmicos para aplicações mecânicas (cerâmicas estruturais) consiste normalmente na síntese do pó, conformação, sinterização e muitas vezes, usinagem. Para aplicações de engenharia que necessitem de tolerância dimensional fechada ou geometria complexa, a usinagem é imprescindível. O sucesso da cerâmica estrutural na maioria das aplicações depende não somente das propriedades do material e do projeto da peça, mas também da qualidade do produto usinado, em termos de acabamento superficial, tensões residuais e defeitos. Os dois fatores freqüentemente citados na literatura como obstáculos ao amplo uso das cerâmicas são a falta de confiabilidade dos componentes de cerâmica e o custo elevado. Jahanmir (1999) e Chand e Guo (2000) citam que a retificação, que é o processo mais utilizado industrialmente na usinagem de cerâmica, pode representar até $90 \%$ do custo total do componente cerâmico. A falta de confiabilidade advém da grande dispersão dos valores de resistência mecânica provocada por defeitos, que podem ter origem no processo de retificação.

Assim como na indústria de processamento metalmecânica, a retificação de cerâmica é realizada normalmente com abundância (elevada vazão e baixa pressão) de fluidos de corte. A função do fluido de corte é reduzir o atrito entre a ferramenta e a peça através da lubrificação (baixas velocidades de corte), remover o calor gerado pelo atrito (altas velocidades de corte), remover os cavacos na interface e proteger o conjunto contra a corrosão. O uso desses fluidos refrigerantes resulta em problemas ecológicos, fisiológicos e econômicos. Desde a última década, a opinião pública, o mercado, as leis e regulamentos têm pressionado as indústrias a considerar os riscos ambientais na manufatura. Uma alternativa é a eliminação total do fluido, através da usinagem a seco. A óbvia vantagem é a eliminação dos riscos à saúde e danos ecológicos, provenientes dos processos de disposição das peças, dos cavacos e do lubrificante em si, bem como dos custos envolvidos. No entanto, estas vantagens são contrapostas pelos problemas provenientes da ausência das funções primárias do fluido refrigerante. Os casos de retificação a seco de cerâmica são extremamente raros na literatura. Entre as abordagens alternativas visando minimizar a quantidade de fluido de corte utilizada na retificação estão a refrigeração otimizada e a técnica da Mínima Quantidade de Lubrificação (MQL). A retificação com refrigeração convencional consiste na utilização de elevadas vazões de fluido de corte à baixa pressão (e conseqüentemente baixa velocidade do fluido de corte). Na refrigeração otimizada, o fluido de corte é acelerado à velocidade igual ou superior à velocidade periférica do rebolo, penetrando na região de corte de maneira mais eficaz. Já a técnica da Mínima
Quantidade de Lubrificação (MQL) implica direcionar uma quantidade mínima de fluido pulverizado através de um jato de ar a elevada velocidade na região de retificação.

O objetivo deste trabalho é avaliar os métodos de lubrirefrigeração, convencional, otimizado e MQL quando da retificação da alumina. Força tangencial específica de corte, rugosidade e relação $\mathrm{G}$ foram os parâmetros de avaliação.

\section{Mecanismos de remoção de material em peças cerâmica}

Os mecanismos de remoção de material podem ser descritos através da interação entre o meio de retificação (grão abrasivo) e a peça a ser retificada. Estes experimentos, por sua vez, podem ser categorizados em dois principais grupos: os experimentos baseados na abordagem de mecânica de fratura aplicada à endentação estática e testes de riscamento com ponta única ou multiponto (grãos abrasivos), envolvendo até experimentos completos de retificação [16].

A abordagem de mecânica de fratura no processo de indentação descreve a interação de um grão abrasivo como um evento, onde o abrasivo penetra a peça na direção normal, enquanto é arrastado na direção lateral. O modelo das trincas induzidas em função da penetração, pode ser observado na Fig. 1.

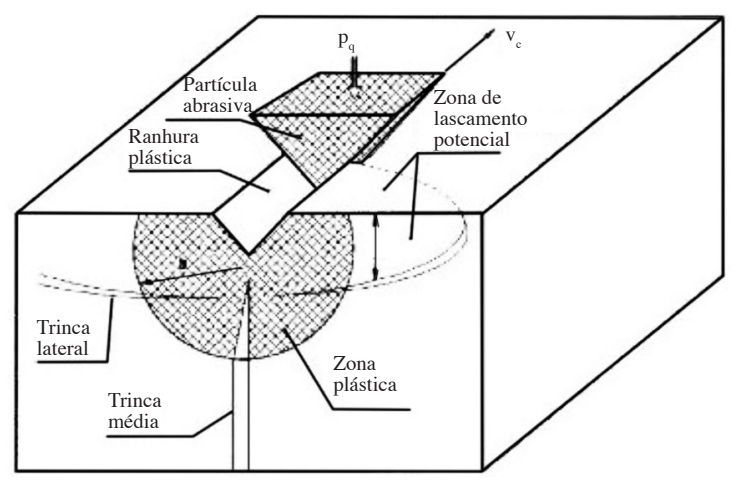

Figura 1: Zona plástica e formação das trincas médias/radiais e laterais devido ao riscamento por um grão abrasivo [17].

[Figure 1: Plastic zone and radial/average crack formation of crack due to scratching by an abrasive grain [17].]

Numa indentação, o contato do indentador com a superfície da cerâmica, inicialmente desenvolve uma zona plástica de pequeno diâmetro, conforme ilustrado na Fig 2a. Em seguida, uma pequena trinca longitudinal (trinca média) inicia-se devido ao campo de tensão desenvolvido (Fig. 2b). A trinca se propaga à medida que a indentação prossegue e aumenta de tamanho (Fig. 2c). Um decréscimo da carga resulta na redução do tamanho ou no fechamento da trinca longitudinal devido às tensões de compressão (Fig. 2d). A redução subseqüente da carga promove a formação de trincas transversais devido às tensões laterais (Fig. 2e). Após a liberação da carga, devido ao campo de tensão residual desenvolvido, o tamanho da trinca lateral aumenta, conduzindo à separação do material na forma de cavacos (Fig. 2f). 

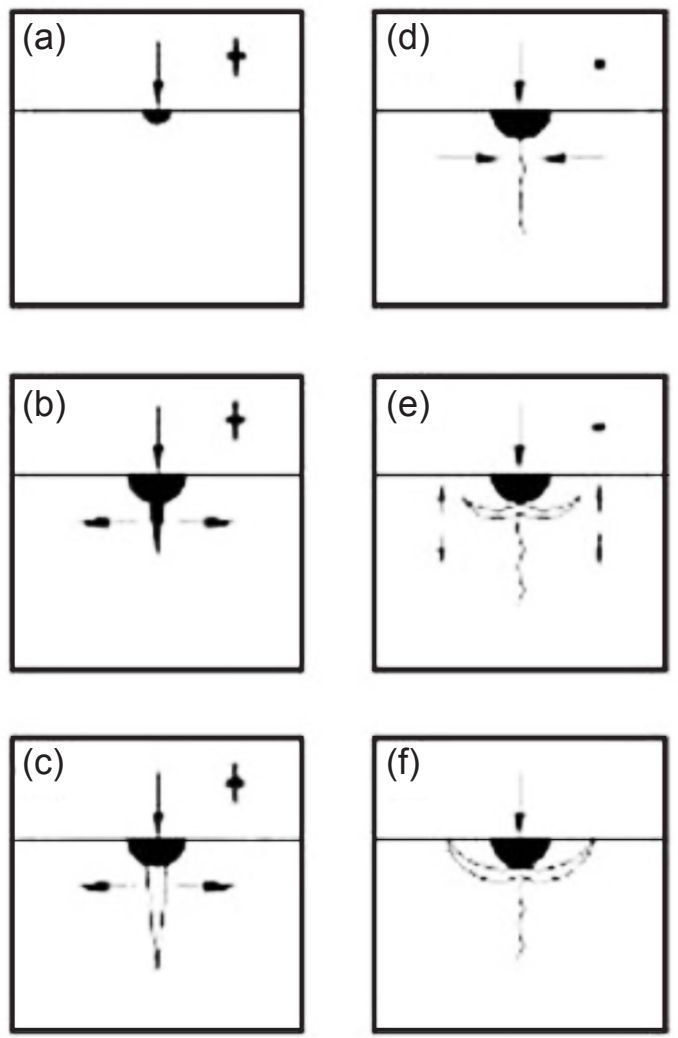

Figura 2: Estágios de formação das trincas através da indentação [13].

[Figure 2: Periods of crack formation through indentation [13].]

As trincas médias que penetram na peça são responsáveis pelo dano à resistência mecânica da peça, enquanto as trincas laterais são responsáveis pela remoção de material. A abordagem de indentação é útil para obter informações fundamentais sobre a evolução da trinca durante a retificação de materiais frágeis, porém falha na captura de interações e influências dos parâmetros de processo [5].

Quando a indentação é realizada com baixa carga, somente uma deformação plástica (permanente) é introduzida na peça. Para a maioria dos materiais frágeis, esta carga limite é relativamente baixa e não depende exclusivamente das propriedades mecânicas do material indentado, mas também da forma do indentador [15].

O mecanismo de remoção de material na retificação de cerâmicas depende do tamanho e da densidade de defeitos tais como falhas, trincas e o tamanho do campo tensionado. Segundo os autores, quando a região onde é aplicada a tensão é menor que o tamanho do defeito, o material é removido principalmente por deformação plástica. Caso contrário, o modo frágil é predominante [10].

\section{Mecanismo de remoção por modo dúctil}

A remoção de material no modo dúctil ocorre na retificação com cargas ou profundidades de corte abaixo do limite para induzir a nucleação de trincas. Neste modo de retificação, o processo de remoção de material não é bem caracterizado e fluxo plástico ou cisalhamento parecem ser descrições mais adequadas [6].

A principal característica do modo dúctil é a ausência de trincas residuais na peça. É consenso na literatura [1] que trincas originadas durante a retificação podem degradar a resistência à ruptura de um componente de cerâmica estrutural. Assim, a resistência mecânica de componentes cerâmicos retificados no modo frágil é muito sensível tanto ao processo de retificação, como à direção de retificação.

Para impedir a formação de trincas na retificação, ou seja, retificar no modo dúctil, duas estratégias são normalmente usadas: o controle da profundidade de corte e o controle da carga por grão abrasivo durante a retificação [9]. A profundidade de corte crítica para a retificação de materiais duros e frágeis no modo dúctil varia de $50 \mathrm{~nm}$ a $1 \mu \mathrm{m}$. O modo dúctil e frágil de retificação pode ocorrer no mesmo material frágil e a transição entre eles pode ser controlada pela configuração dos parâmetros de processo (avanço e fluido de corte). Em seu trabalho, ele reporta um modo intermediário entre o frágil e o dúctil, que ele chama de retificação por microtrincamento ou retificação parcialmente dúctil [2].

\section{Transição entre o modo frágil e dúctil}

A transição entre os modos de remoção de material pode ser compreendida baseada no conceito de escala. Conforme o tamanho ou escala (volume) da interação entre o grão abrasivo e a superfície da peça diminui, há um limite onde a fratura do material da peça é substituída pela deformação plástica. Para interações de pequena escala na maioria dos materiais frágeis, é mais favorável energeticamente deformar-se do que criar duas novas superfícies por fratura. A escala limite em que ocorre esta transição é uma função das propriedades intrínsecas que determinam a fratura e deformação plástica do material [9].

A escala de retificação baseado na densidade de defeitos do material a ser retificado em três regiões: maior que a escala de micrômetro - nesta região, microtrincas e discordâncias estão normalmente incluídas no campo tensionado. Conforme aumenta a escala de retificação, aumenta o número de defeitos no campo tensionado e diminui a energia necessária para propagação de trincas (modo frágil); escala de micrômetro a sub-micrômetro - a energia para fratura não diminui nesta região conforme o campo tensionado diminui porque as microtrincas raramente ocorrem no campo tensionado. O mecanismo de remoção de material tende a mudar de frágil para dúctil; escala de sub-micrômetro para nanômetro - nesta região a retificação é realizada numa superfície livre de defeitos. A energia para fratura tende para o mesmo nível de energia necessária para deformação plástica. Esses valores são ocasionalmente afetados pela temperatura ambiente [8].

O critério da espessura de corte crítica, que estabelece que se a espessura máxima do cavaco obtido na retificação é menor que a espessura de corte crítica que produz trincas no material, então a retificação ocorrerá no regime dúctil [16]. 
A espessura máxima do cavaco é dada pela Eq. A:

$$
\mathrm{d}_{\mathrm{c}}=\left(\frac{\mathrm{E}}{\mathrm{H}}\right)\left(\frac{\mathrm{K}_{\mathrm{IC}}}{\mathrm{H}}\right)^{2}
$$

O efeito do $\mathrm{pH}$ do fluido de corte sobre a dureza da cerâmica e a conseqüente alteração na transição do modo de remoção. A dureza seria afetada pela movimentação de discordâncias. As variações na caracterização após a retificação (resistência mecânica e rugosidade), bem como parâmetros de processo (forças de retificação) em função da dureza/pH [4].

Na Fig. 3 é introduzido um gráfico que relaciona a dureza da alumina em função do $\mathrm{pH}$ e potencial zeta do fluido de corte. O potencial zeta representa o potencial no ponto entre a porção difusa da dupla camada elétrica, que é formada espontaneamente na interface com um eletrólito [3]. Ele representa o potencial eletrostático gerado pelo acúmulo de íons na superfície de uma partícula em contato com um eletrólito.

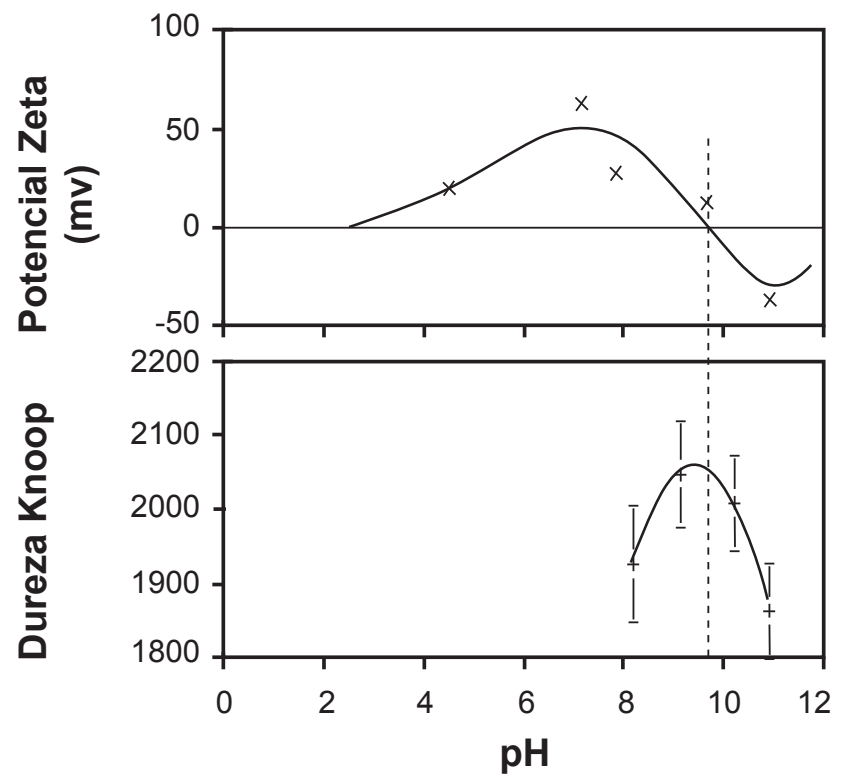

Figura 3: Variação da dureza em função do $\mathrm{pH}$ e do potencial zeta do fluido de corte [3].

[Figure 3: Variation of hardness related to $\mathrm{pH}$ and zeta potential of the cutting fluid [3].]

\section{Mecanismo de fratura frágil}

Alguns modelos foram propostos para descrever o processo frágil de remoção de material durante a usinagem abrasiva de cerâmica. A formação e propagação de uma trinca em analogia ao processo de indentação [9]. Posteriormente esse modelo foi detalhado [17]. Segundo o modelo, as trincas laterais são responsáveis pela remoção de material e seu tamanho está relacionado com a carga e as propriedades do material através da equação $B$ :

$$
\mathrm{V}=\alpha \mathrm{P}^{\frac{9}{8}}\left(\frac{\mathrm{E}}{\mathrm{H}}\right)^{\frac{4}{5}}\left(\mathrm{~K}_{\mathrm{IC}}^{\frac{1}{2}} \mathrm{H}^{\frac{5}{8}}\right)
$$

na qual $\mathrm{V}=$ volume de material removido por unidade de distância de deslizamento; $\alpha=$ constante independente do material; $\mathrm{P}=$ força normal aplicada aos grãos abrasivos.

A equação para descrever a força crítica para ativação do processo de remoção por trinca lateral [9]:

$$
\mathrm{P}^{*}=\xi . \mathrm{f}\left(\frac{\mathrm{E}}{\mathrm{H}}\right) \cdot\left(\mathrm{K}_{\mathrm{IC}}{ }^{4} \mathrm{H}^{3}\right)
$$

ou

$$
\mathrm{P}^{*}=\xi \cdot \mathrm{f}\left(\frac{\mathrm{E}}{\mathrm{H}}\right) \cdot\left[\mathrm{K}_{\mathrm{IC}}\left(\frac{1}{\mathrm{~B}}\right)^{3}\right]
$$

nas quais $\xi=$ constante adimensional; $f(\mathrm{E} / \mathrm{H})=$ função fraca cujo produto com $\xi$ equivale a $2.10^{5}$ para indentação Vickers.

A equação D indica uma forte influência do índice de fragilidade na carga crítica para ativação da fratura lateral. Um outro modelo é baseado na formação e extensão de um grande número de microtrincas distribuídas na região tensionada. Essas trincas interagem e promovem a remoção de material. A hipótese de retificação em regime de pulverização. Segundo os autores, a pulverização é conseqüência do microtrincamento intragranular ou transgranular devido ao campo de tensões de cisalhamento sobrepostas por tensões compressivas hidrostáticas, induzidas pelos grãos abrasivos durante a retificação. Os dados obtidos pelos autores sugerem que a fragilidade desempenha um papel significativo neste processo.

Os grãos pulverizados formam uma camada superficial de menos de $10 \mu \mathrm{m}$, localizada imediatamente acima da região de microtrincamento, conforme apresentado na Fig. 4. A espessura desta camada estaria correlacionada com a profundidade de corte e o tamanho do grão abrasivo [2].

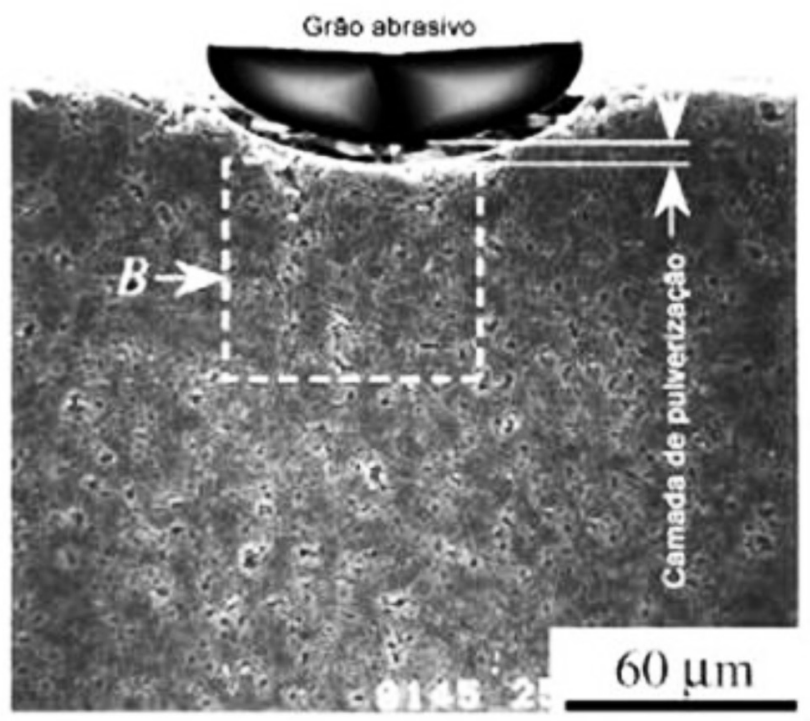

Figura 4: Fotografia apresentando a camada onde ocorre a pulverização [2].

[Figure 4: Photograph presenting the layer where pulverization occurs [2].] 
Embora os processos de remoção através de deformação plástica e fratura frágil possam ocorrer na retificação de cerâmicas policristalinas, o processo de microfratura e remoção em escala de grão são mais comuns. O processo de microfratura é a remoção através da propagação de trincas ao longo dos contornos de grãos [9].

\section{METODOLOGIA DE EXPERIMENTAÇÃO}

Para a avaliação do desempenho dos métodos de lubrirefrigeração, foram realizados testes de retificação com uma retificadora plana tangencial $1055 \mathrm{E}$ da Sulmecânica. Os ensaios foram realizados com rebolo diamantado de liga resinóide de diâmetro $350 \mathrm{~mm}$, largura $15 \mathrm{~mm}$, dureza da liga $\mathrm{N}$, concentração 50 e tamanho de grão $126 \mu \mathrm{m}$. Os corpos-deprova consistiram de barras retangulares, nas dimensões 109 $\mathrm{mm} \times 34 \mathrm{~mm}$ × $9 \mathrm{~mm}$, de uma alumina comercial, composta por $96 \%$ de óxido de alumínio e $4 \%$ de óxidos fundentes como $\mathrm{SiO}_{2}, \mathrm{CaO}$ e $\mathrm{MgO}$. Este material apresentou densidade aparente $3,701 \pm 0,002 \mathrm{~g} / \mathrm{cm}^{3}$. As demais propriedades deste material estão na Tabela I.

Tabela I - Propriedades do material ensaiado. [Table I - Properties of the tested material.]

\begin{tabular}{lcc}
\hline \multicolumn{1}{c}{ Propriedade } & Norma & Valor \\
\hline Resistências à & ASTM & $279 \pm 11 \mathrm{MPa}$ \\
Flexão 4 pontos & C1161-02 & \\
Tenacidade à & ASTM & $3,18 \pm 0,07 \mathrm{MPa} . \mathrm{m}^{1 / 2}$ \\
Fratura & C1421-01 & \\
Módulo de & JIOS R1602- & $312 \pm 4 \mathrm{GPa}$ \\
Elasticidade & 1986 & \\
Microdureza & JIS R1610- & $1339 \pm 47 \mathrm{HV} 1$ \\
Vickers & 1991 & \\
Tamanho médio & ASTM E & $1,57 \mu \mathrm{m}$ \\
de grão & $1382-97$ & \\
\hline
\end{tabular}

Utilizou-se nesta pesquisa três métodos distintos de lubrirefrigeração, sendo que em cada um deles empregou-se um bocal distinto. O método de lubri-refrigeração convencional (Fig. 5a), caracterizado pela aplicação de fluido de corte a alta vazão e baixa pressão, foi realizado através de um bocal de seção retangular de dimensões $49 \mathrm{~mm}$ x $3 \mathrm{~mm}$ e área seccional de $147 \mathrm{~mm}^{2}$. Este bocal trabalhou com vazão de $27,5 \mathrm{~L} / \mathrm{min}$, pressão inferior a $0,2 \mathrm{kgf} / \mathrm{cm}^{2}$ e velocidade do fluido de corte de $3 \mathrm{~m} / \mathrm{s}$. No método de lubri-refrigeração otimizada utilizouse um bocal com uma pequena área seccional quadrada (13,5 $\mathrm{mm} \times 0,7 \mathrm{~mm}$ ). Na Fig. $5 \mathrm{~b}$ este bocal é apresentado. Ele foi desenvolvido de tal forma que a velocidade do fluido de corte era igual à velocidade periférica do rebolo, permitindo uma lubrirefrigeração mais eficiente [18]. Nesta condição, trabalhou-se com vazão $17 \mathrm{~L} / \mathrm{min}$, pressão $6 \mathrm{kgf} / \mathrm{cm}^{2}$ e velocidade do fluido $30 \mathrm{~m} / \mathrm{s}$. A Fig. 5 b ilustra o posicionamento do bocal durante os ensaios de retificação.
As propriedades mecânicas que um corpo cerâmico possui têm influência direta de sua composição química e forma de fabricação. A seguir são estudados alguns dos principais fatores que são essenciais para garantir boas propriedades ao material cerâmico fabricado.
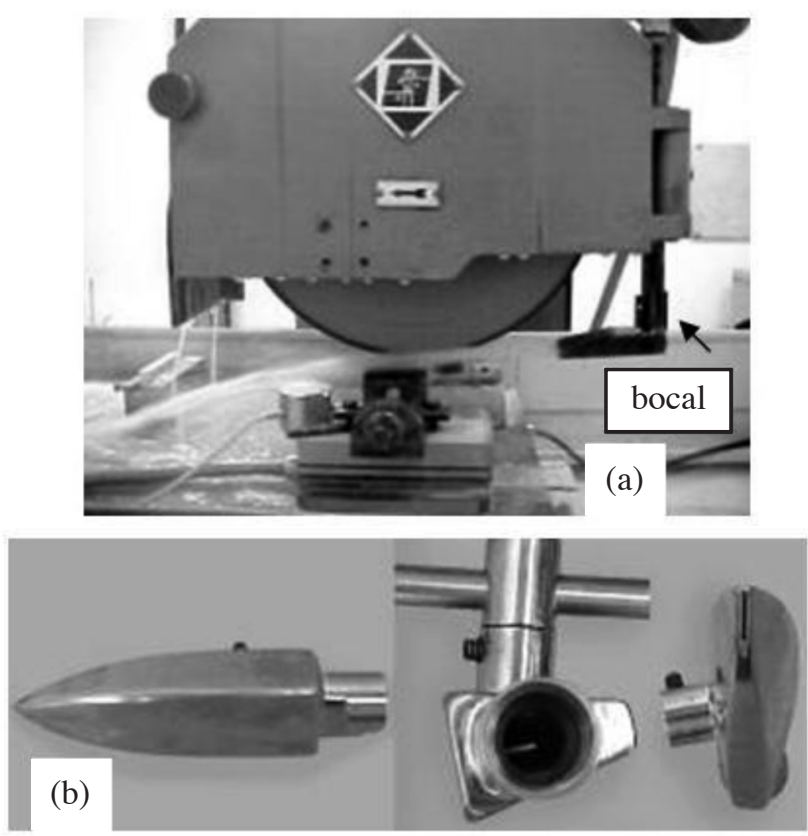

Figura 5: a - Bocal convencional; b - Bocal otimizado. [Figure 5: a - Conventional nozzle; $b$ - Optimized nozzle.]

Para a execução da Mínima Quantidade de Lubrificação (MQL) foi utilizado um bocal específico que permitia melhor eficácia de lubrificação na zona de corte, semelhante ao utilizado na retificação com rebolos de CBN [11]. A vazão de ar foi determinada considerando uma pressão de $8 \mathrm{kgf} / \mathrm{cm}^{2}$ e a velocidade do ar comprimido também igual à velocidade periférica do rebolo [18]. A vazão ideal encontrada foi de $26,6 \mathrm{~m}^{3} / \mathrm{h}(450 \mathrm{~L} / \mathrm{min})$. A vazão de óleo determinada em ensaio preliminar foi $80 \mathrm{~mL} / \mathrm{h}$. O controle das vazões do óleo e do ar foi feito por registros de agulha existente no interior do equipamento utilizado. O fluido de corte utilizado nas condições convencional e otimizado foi um óleo solúvel sintético à base de éster de poliglicol, com concentração entre $4 \%$ a $6 \%$ e pH entre 8,5 e 9,5 . Na condição de mínima lubrificação foi testado fluido de corte integral (óleo) sem diluição. A Tabela II apresenta o resumo das condições de aplicação dos fluidos.

O trabalho consistiu em 3 ensaios de retificação, 1 para cada método de refrigeração. A profundidade de corte foi $0,01 \mathrm{~mm}$. Todos os ensaios foram realizados com velocidade de corte $\left(\mathrm{V}_{\mathrm{s}}\right) 30 \mathrm{~m} / \mathrm{s}$ e velocidade da mesa da retificadora $\left(\mathrm{V}_{\mathrm{w}}\right)$ 2,3 m/min. Na Tabela III são apresentados os parâmetros de retificação em função da profundidade de corte.

A medição da força tangencial de corte foi realizada através de um dinamômetro piezelétrico Kistler 9257BA e uma unidade de controle 5233A1. Este dinamômetro consiste de quatro sensores de força de três componentes ( $\mathrm{x}$, 
Tabela II - Resumo dos parâmetros relacionados à aplicação do fluido de corte.

[Table II - Summary of the parameters related to the application of the cutting fluid.]

\begin{tabular}{ccccc}
\hline Método de lubri-refrigeração & Convencional & Otimizado & $\begin{array}{c}\text { MQL } \\
\text { Ar }\end{array}$ & Óleo \\
\hline Vazão [L/min] & 27,5 & 17 & 450 & 0,0013 \\
Pressão $\left[\mathrm{kgf} / \mathrm{cm}^{2}\right]$ & $<0,2$ & 6 & 8 & \multirow{2}{*}{30} \\
Velocidade do fluído [m/s] & 3 & 30 & 30 & \\
\hline
\end{tabular}

Tabela III - Parâmetros de retificação adotados nos ensaios. [Table III - Adopted grinding parameters in the experiments.]

\begin{tabular}{ccccc}
\hline $\begin{array}{c}\mathrm{a} \\
(\mathrm{mm})\end{array}$ & $\begin{array}{c}\mathrm{h}_{\mathrm{eq}} \\
(\mu \mathrm{m})\end{array}$ & $\begin{array}{c}\mathrm{l}_{\mathrm{c}} \\
(\mathrm{mm})\end{array}$ & $\begin{array}{c}\mathrm{Q}_{\mathrm{w}} \\
\left(\mathrm{mm}^{3} / \mathrm{min}\right)\end{array}$ & $\begin{array}{c}\mathrm{Q}^{\prime}{ }^{\mathrm{w}} \\
\left(\mathrm{mm}^{3} / \mathrm{mm} . \mathrm{s}\right)\end{array}$ \\
\hline 0,01 & 0,77 & 1,87 & 207 & 0,38 \\
\hline
\end{tabular}

y e z). O gerenciamento destes dados também foi realizado via LabView.

O desgaste do rebolo foi determinado com o propósito de avaliar a relação $\mathrm{G}$, ou seja, a relação entre o volume de material removido durante a retificação e o volume de rebolo desgastado. O corpo de prova possuía menor largura $(8 \mathrm{~mm})$ que o rebolo $(15 \mathrm{~mm})$ e assim, desgastou somente a faixa de contato com o rebolo, criando um rebaixo na região central do rebolo. Este perfil de baixo relevo foi copiado numa peça retificada para medir a diferença de altura entre a região desgastada pelo rebolo (centro) e a região sem contato com o rebolo. O perfil impresso na peça retificada foi traçado através de um medidor de perfil, rugosidade e forma Taylor Hobson Form Talysurf Intra series I120. O volume de material removido dos corpos de prova retificados foi obtido através de medições antes e depois do ensaio, com paquímetro digital Tesa, de resolução $0,01 \mathrm{~mm}$. O volume de material removido por ensaio foi de $20.000 \mathrm{~mm}^{3}$. Para efetuar as medições de rugosidade das superfícies retificadas, representada pelo parâmetro Ra, o rugosímetro foi ajustado para um comprimento de amostragem (cut-off) de $0,8 \mathrm{~mm}$. Vale salientar que a medição foi realizada na direção perpendicular à superfície de retificação e que o raio da ponta do diamante do medidor de rugosidade foi de $2 \mu \mathrm{m}$.

\section{RESULTADOS E DISCUSSÃO}

\section{Resultados de força tangencial especifica de corte}

Os métodos de lubri-refrigeração testados apresentaram valores de força específíca de corte similares, mantendo-se estável ao longo da execução dos ensaios. No ensaio com lubri-refrigeração através de MQL, ao final deste ensaio ocorreu a queima do ligante do rebolo. A força tangencial obtida foi muito inferior, talvez provocada pelo desgaste acentuado do rebolo. A hipótese de degradação do rebolo é confirmada pela acentuada queda da força tangencial

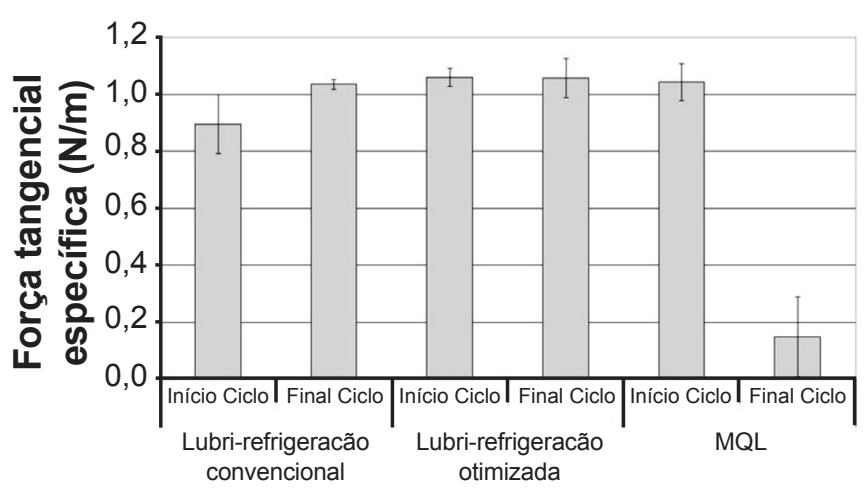

Figura 6: Comparativo entre as forças tangenciais de corte obtidas com os três métodos de lubri-refrigeração, com profundidade de corte de 0,01 mm e "spark-out".

[Figure 6: Comparative between tangential forces with the tree methods of lubri-refrigeration, using depth of cut of $0.01 \mathrm{~mm}$ and "spark-out".]

entre o início do ensaio (que apresentava valores próximos à condição convencional e otimizada) e o final, conforme ilustrado na Fig. 6.

\section{Resultados de rugosidade}

No ensaio com MQL a rugosidade apresentou acentuado acréscimo comparando os valores obtidos antes e depois da retificação, Fig. 7. O aumento da rugosidade pode ser explicado pela degradação do rebolo em função da queima

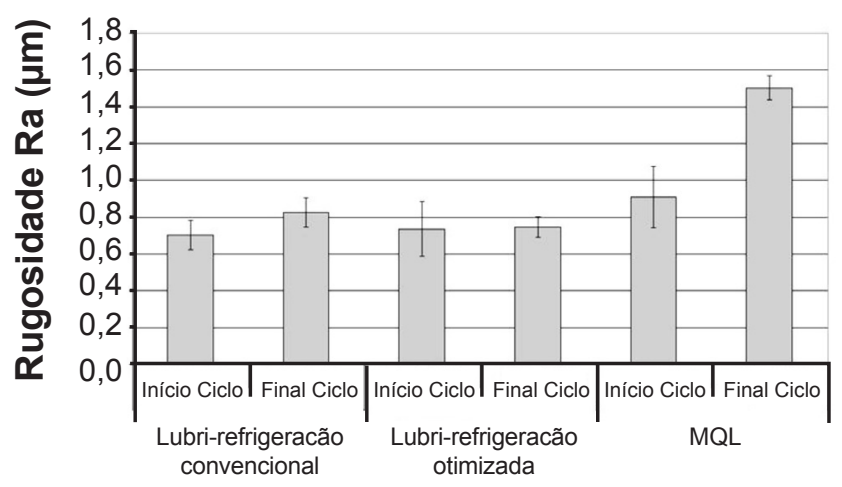

Figura 7: Valores de rugosidade Ra obtidos para cada método de lubri-refrigeração, com profundidade de corte de $0,01 \mathrm{~mm}$ e "sparkout".

[Figure 7: Values of Ra roughness for each method of lubrirefrigeration, with depth of cut of $0.01 \mathrm{~mm}$ and "spark-out".] 
do ligante e de possíveis tensões induzidas por choque térmico devido à baixa condutividade térmica da cerâmica e da corrente de ar.

\section{Resultados de desgaste radial}

Os ensaios realizados com o método convencional apresentaram maior relação $G$ que aqueles realizados com a lubri-refrigeração otimizada. Comparando os três métodos na mesma condição, de acordo com a Fig. 8, o método de lubri-refrigeração através do MQL apresentou menor relação $\mathrm{G}$ (maior desgaste), devido à degração térmica do ligante que acelerou o macrodesgaste através da remoção de liga e do diamante.

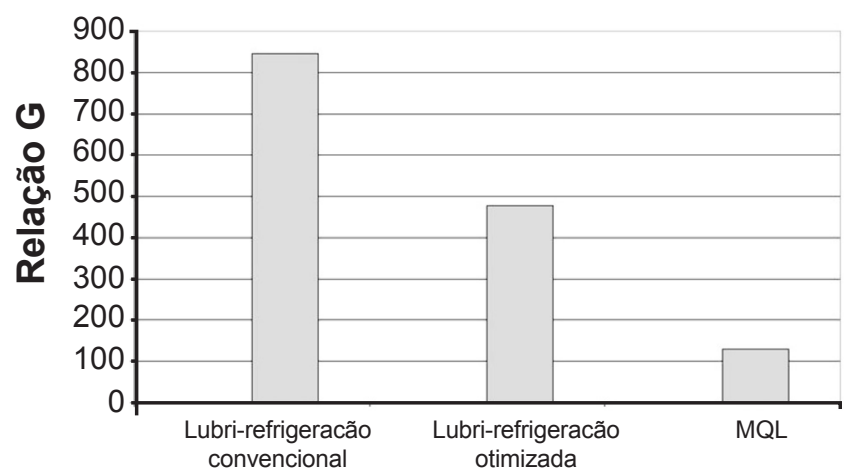

Figura 8: Valores de rugosidade Ra obtidos para cada método de lubri-refrigeração, com profundidade de corte de $0,01 \mathrm{~mm}$ e "spark-out".

[Figure 8: Values of Ra roughness for each method of lubrirefrigeration, with depth of cut of $0.01 \mathrm{~mm}$ and "spark-out".]

\section{CONCLUSÕES}

Aconfiguração do bocal proposto para a lubri-refrigeração otimizada produziu resultados estatisticamente equivalentes aos obtidos com a técnica convencional, empregando uma menor quantidade de fluido refrigerante. A técnica de $\mathrm{MQL}$ não é viável na retificação de cerâmica com as condições descritas nesse trabalho (rebolo resinóide, profundidade de corte de $0,01 \mathrm{~mm}$ e "spark-out"). A vazão de ar de $450 \mathrm{~L} / \mathrm{min}$ e de lubrificante de $70 \mathrm{~mL} / \mathrm{h}$ foi insuficiente para promover a refrigeração na área de retificação, provocando a degradação térmica da liga do rebolo, prejudicando a rugosidade da peça e acelerando o desgaste do rebolo. Além disso, o lubrificante formou uma pasta com os cavacos removidos na retificação, dificultando a remoção destes cavacos da área de corte. A força tangencial de retificação manteve-se estável (variação entre o início e final do ensaio) durante a retificação com profundidade de corte de $0,01 \mathrm{~mm}$.

\section{AGRADECIMENTOS}

À FAPESP (Fundação de Amparo à Pesquisa do Estado de S. Paulo) e ao IFM (Instituto Fábrica do Milênio) pelo suporte a esta pesquisa e à UNESP.

\section{REFERÊNCIAS}

[1] B. Mikijelj, R. Allor, Effects of machining on $\mathrm{Si}_{3} \mathrm{~N}_{4}$ strength, Am. Ceram. Soc. Bull. 82, 4 (2003) 57.

[2] B. Zhang, X. L. Zheng, H. Tokura, M. Yoshikawa, Grinding induced damage in ceramics, J. Mater. Proc. Technol. 132 (2003) 353.

[3] D. W. Alley, O. F. Devereux, Coolant $\mathrm{pH}$ control for optimum ceramic grinding, J. Mater. Sci. 37 (2002) 5135.

[4] D. W. Alley, O. F. Devereux, Coolant pH control for optimum ceramic grinding, J. Mater. Sci. 38 (2003) 1353.

[5] G. Subhash, J. E. Loukus, S. M. Pandit, Application of data dependent systems approach for evaluation of fracture modes during a single-grit scratching, Mechanics Mater. 34 (2002) 25.

[6] H. K. Tönshoff, T. Lierse, I. Inasaki, Grinding of advanced ceramics, in S. Jahanmir, M. Ramulu, P. Koshy, Machining Ceramics Composites, Marcel Dekker, EUA (1999) Cap. 3.

[7] H. P. Kirchner, T. J. Larchuk, J. C. Conway, Crushing as a mechanism of material removal during abrasive machining, J. Am. Ceram. Soc. 68, 4 (1985) 209.

[8] I. Marinescu, M. Pruteanu, Deformation and fracture of ceramics materials, in I. Marinescu, H. K. Tönshoff, I. Inasaki, Handbook of Ceramic Grinding and Polishing, Noyes Publ., New Jersey, EUA (1998) Cap. 2.

[9] K. L. Blaedel, J. S. Taylor, C. J. Evans, Ductile-regime grinding of brittle materials, in S. Jahanmir, M. Ramulu, P. Koshy, Machining Ceramics Composites, Marcel Dekker, EUA (1999) Cap. 5.

[10] K. Ramesh, S. H. Yeo, S. Gowri, L. Zhou, Experimental evaluation of super high-speed grinding of advanced ceramics, Int. J. Adv. Manuf. Technol. 17 (2001) 87.

[11] L. R. Silva, E. C. Bianchi, R. Y. Fusse, R. E. Catai, T. V. França, P. R. Aguiar, Analysis of surface integrity for minimum quantity lubricant - MQL in grinding, Int. J. Machine Tools Manuf. 47, 2 (2007) 412.

[12] Z. W. Zhong, Ductile or partial ductile mode machining of brittle materials, Int. J. Adv. Manuf. Technol. 21 (2003) 579.

[13] B. R. Lawn, Fracture of brittle solids, p.257, 2a edição, Cambridge, Cambridge University Press, 1993.

[14] R. H. Chand, C. Guo, Machining technical ceramics: the cost factor, Ceramic Ind. 150, 7 (2000) 31-32; C. C. Chang, A. Z. Szeri, A thermal analysis of grinding, Wear 216 (1998) 77. [15] S. Jahanmir, H. K. Xu, L. K. Ives, Mechanisms of material removal in abrasive machining of ceramics, in S. Jahanmir, M. Ramulu, P. Koshy, Machining Ceramics Composites, Marcel Dekker, EUA (1999) Cap. 2.

[16] S. X. Liang, Grinding of ceramics: mechanisms, forces and coolant evaluation, 113 f. M.Sc. Diss., Univ. Connecticut, EUA (1992).

[17] S. Malkin, T. W. Hwang, Grinding mechanism for ceramics, Annals CIRP 45 (1996) 569.

[18] J. A. Webster, Selection of coolant type and application technique in grinding. Supergrind (1995) 205.

(Rec. 19/03/2009, Ac. 07/08/2009) 